Article

\title{
Electric Field Excitation Suppression in Cold Atoms
}

\author{
Jianing Han *, Juliet Mitchell and Morgan Umstead \\ Physics Department, University of South Alabama, Mobile, AL 36688, USA; jem1622@jagmail.southalabama.edu \\ (J.M.); mfu1521@jagmail.southalabama.edu (M.U.) \\ * Correspondence: jhan@southalabama.edu
}

Received: 21 June 2020; Accepted: 18 August 2020; Published: 20 August 2020

\begin{abstract}
In this article, the atom excitation suppression is studied in two mechanisms. The first mechanism for excitation suppression is caused by an external DC electric field. The second mechanism is due to the energy shift caused by an electric field generated by free charges, which are created by ionizing atoms. The latter mechanism is known as the Coulomb blockade. Here, the Coulomb forces originate from ions created by ionizing atoms with a UV laser. The interaction, which causes the suppression, is treated theoretically as dipole-charge interactions. In the model, the charge is an ion, and the dipole is an atom. From measurements, we use ${ }^{85} \mathrm{Rb}$ atoms. The valence electron and the ion core are the two poles of an electric dipole. The interaction potential energy between the ion and the atom is proportional to $\frac{1}{R^{2}}$, and the frequency shift caused by this interaction is proportional to $\frac{1}{R^{4}}$, where $R$ is the distance between the ion and the dipole considered. This research is motivated by potential applications for quantum information storage, remote control, creating hot plasmas using cold atoms, as well as electronic devices.
\end{abstract}

Keywords: coulomb blockade; cold atoms; quantum information; dipoles

PACS: $34.20 . G j ;$ 34.50.Fa; 03.65.-w

\section{Introduction}

We first study the ground state excitation suppression caused by the Stark shift of the atoms by adding an external DC electric field. We then study the Coulomb field effect on excitation suppression. This can be called Coulomb blockade. Coulomb blockade was originally studied in electronic devices. The basic idea is that, due to the Coulomb interaction between the charges in the electronic device, the conductivity of the device is affected [1-4]. In this article, we study the Coulomb blockade in a cold dilute atomic gas. Unlike the Rydberg blockade induced by ions [5], we focus on the blockade on $5 \mathrm{P}$ atoms. We purposely create charges inside the atomic gas, and we then study the energy shift caused by those charges, which in turn affects the conductivity of such gases. The conductivity in such systems can be estimated by measuring the time of flight of the ionized excited atoms. In this article, we report the excitation suppression of the system caused by an external electric field through imaging the cold atoms.

Very similar to the dipole-blockade studied in Rydberg gases, here the energy shift is caused by charges instead of dipoles. Dipole-blockade, the excitation suppression due to the energy shift caused by neighboring dipoles, has been proposed and experimentally studied due to its potential applications as quantum gates [6-15]. The interactions in dipole-blockade are caused by dipole-dipole interactions. Each dipole there is a Rydberg atom since Rydberg atoms have large electric dipole moments. The excited electron and the ion core in a Rydberg atom $[16,17]$ are the two poles of an electric dipole. In this article, we study the Coulomb blockade, or dipole-charge interactions, in the lower level atoms. Here, we treat a lower level atom, a $5^{2} P_{3 / 2}$ atom, as a dipole. Those atoms do 
not have permanent dipoles; however, under an external electric field, such atoms will be polarized, or an induced dipole will be created. The advantage of Coulomb blockade in regard to capabilities, compared to the dipole blockade, is that it can work in an extensive range due to the stronger interaction strength between charges and dipoles. Specifically, we create ions and study ion-atom interactions or dipole-charge interactions. Such states not only can be used for quantum gates but also for quantum information sciences. For example, they can be used to store quantum information. Moreover, the research reported can be used to create plasmas with a particular temperature [18-21].

In this work, two ways of suppressing ground state excitation are described separately. Section 2 discusses the simple case of an externally applied DC electric field. Section 3 treats the suppression of excitation due to Coulomb blockade arising from ion-charge interaction. Theoretical expectations are compared with experimental results. Finally, in Section 4, we conclude with a summary of the main results of this study.

\section{DC Field Suppression}

\subsection{Experiment}

The experimental apparatus is very similar to experimental setups reported earlier [22]. A Magneto-Optical Trap (MOT) is created by three pairs of retro-reflected beams. A repump laser is intersected with the three pairs of beams at the center of the vacuum chamber. An electric field is created between two pairs of rods. Four rods are located at the four corners of a square with the MOT at the center of the square [23]. The side length of the square is one inch. Two of the rods are connected, and a high voltage is added on this pair of rods, which can be used to collect ions. The other pair is connected and grounded. Figure $1 \mathrm{a}$ is the schematic plot of the MOT and the electric field created. Figure $1 \mathrm{~b}$ shows one atom in the MOT polarized in the electric field direction. The data are taken by taking the images of the MOT. Figure 1c shows two images taken at center fields $0 \mathrm{~V} / \mathrm{cm}$ and $1100 \mathrm{~V} / \mathrm{cm}$, respectively (or $0 \mathrm{~V}$ and $2800 \mathrm{~V}$ on one pair of rods and the other pair is grounded). It is shown that, at a higher voltage or a higher field, the image intensity is lower, which indicates a lower number of excited atoms, or excitation suppression. The temperature of the atoms in the MOT is about $300 \mu \mathrm{K}$. We take the images of the MOT by a stingray camera at a $5 \mathrm{~Hz}$ repetition rate through a Vimba Viewer program (https:/ / www.alliedvision.com/en/products/software.html). The images are then stored in a computer, integrated, and processed. The relative number of atoms is calculated by integrating the MOT image files. In this experiment, we vary the electric field strength and monitor the relative number of atoms in the MOT.

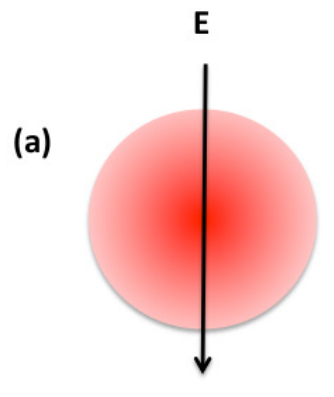

(b)

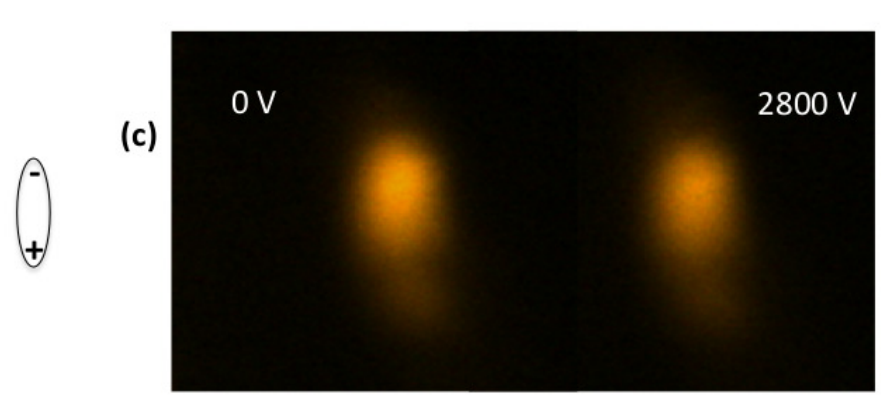

Figure 1. (a) The MOT at the center of the vacuum chamber and the electric field is added across the MOT as shown by E. (b) One atom in the MOT is polarized under the electric field; (c) two images taken at a zero field and $1100 \mathrm{~V} / \mathrm{cm}$ or $2800 \mathrm{~V}$ on two pairs of rods which are 1 inch apart. Each image is 184 pixels $\times 208$ pixels [22]. 


\subsection{Results and Discussion}

Figure 2 shows two sets of data taken at two different densities or numbers of atoms. The densities are changed by attenuating the repump laser beam and confirmed by the imaging. In this case, the sizes of the MOT under those two different densities are approximately the same. The vertical axis is the relative number of atoms, which can be estimated by collecting the fluorescence from the MOT. All the data points in Figure 2 are taken on the same day. The total number of atoms varies from day to day. The data taken on different days show the same trend, and by switching fields on and off, clear suppression has been repeatedly observed. The field that was constant which each image was taken. The data were taken either in the forward direction, increasing the voltage, or in the backward direction, decreasing the voltage. In both cases, similar excitation suppression has been observed.

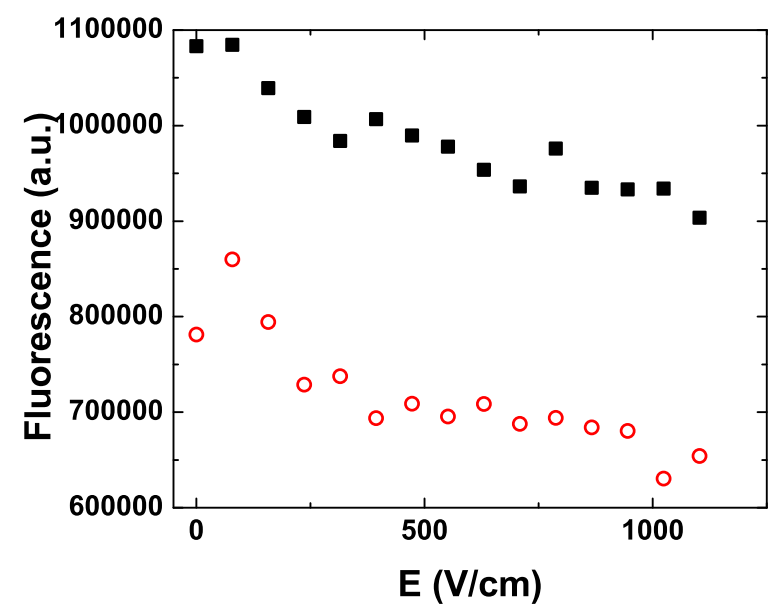

Figure 2. The relative number of atoms in the MOT vs. the electric field added.

We assume this suppression is caused by the Stark effect. We have calculated the Stark effect for the trapping transition and repump transition. The Hamiltonian that we used to calculate the Stark effect is the following [24]:

$$
H_{\text {Stark }}=-\frac{1}{2} \alpha_{0} E_{z}^{2}-\frac{1}{2} \alpha_{2} E_{z}^{2} \frac{3 J_{z}^{2}-J(J+1)}{J(2 J-1)} .
$$

We define the electric field direction as the $z$-direction, and the magnitude of the electric field added is $E_{z} \cdot \alpha_{0}$ and $\alpha_{2}$ are the scalar and tensor polarizabilities. $J$ and $J_{z}$ are the total electron angular momentum state and the projection of the total electron angular momentum state in the $\mathrm{z}$ axis. In this calculation, the ground state scalar polarizability that we used is $\alpha_{0}=0.0794 \mathrm{~h} \mathrm{~Hz} /(\mathrm{V} / \mathrm{cm})^{2}$ for $5^{2} \mathrm{~S}_{1 / 2}$ states. The $5^{2} \mathrm{P}_{3 / 2}$ scalar polarizability is $\alpha_{0}=0.2134 \mathrm{~h} \mathrm{~Hz} /(\mathrm{V} / \mathrm{cm})^{2}$ and the $5^{2} \mathrm{P}_{3 / 2}$ tensor polarizability is $\alpha_{2}=-0.0406 \mathrm{~h} \mathrm{~Hz} /(\mathrm{V} / \mathrm{cm})^{2}$, where $h$ is the Planck's constant [24].

Figure 3 shows the energy shift caused by the Stark effect. In this plot, we calculated the energy difference between the $5^{2} P_{3 / 2} \mathrm{~F}=4$ state and the $5^{2} S_{1 / 2} \mathrm{~F}=3$ state, or the trapping transition as shown in Figure $3 a$, relative to the energy difference between those two states at zero electric fields, $E$ :

$$
E=\left(E_{5^{2} P_{3 / 2}, F=4, D C}-E_{5^{2} S_{1 / 2}, F=3, D C}\right)-\left(E_{5^{2} P_{3 / 2}, F=4}-E_{5^{2} S_{1 / 2}, F=3}\right) .
$$

where $E_{5^{2} P_{3 / 2}, F=4}$ is the energy of the $5^{2} P_{3 / 2}, F=4$ state. $E_{5^{2} P_{3 / 2}, F=4, D C}$ is the energy of the same state under an external DC electric field. The different energy levels correspond to the different $F_{z}$ states, where $F_{z}$ is the operator of the projection of the total atomic angular momentum, $F=I+J$, in the $z$-direction. $I$ is the nuclear angular momentum. For example, the top state is the energy difference 
between $5^{2} P_{3 / 2}, F=4, m_{F}=4$ and $5^{2} S_{1 / 2}, F=3$ state calculated from Equation (2), where $m_{F}$ is the absolute value of $F_{z}$. Since different $F_{z}$ states of $5^{2} S_{1 / 2}, F=3$ at the field strength considered are approximately degenerate, no $F_{z}$ state is specified for $5^{2} S_{1 / 2}, F=3$. Similar to the trapping transition, the corresponding repump transition is also calculated, as shown in Figure $3 \mathrm{~b}$. The different levels again correspond to different $F_{z}$ states. It is shown that the shift (the span on the right side of Figure $3 \mathrm{a}, \mathrm{b}$ ) of the trapping transition at about $500 \mathrm{~V} / \mathrm{cm}$, which corresponds to the voltage $1270 \mathrm{~V}$ on the two pairs of rods in our case, is about $10 \mathrm{kHz}$, and the span of the repump transition at the same voltage is about $3 \mathrm{kHz}$. The linewidth of the atomic transition is on the order of $\mathrm{kHz}$ or less. The line width of the trapping laser or repump laser is about $1 \mathrm{MHz}$. The fact that the frequency span at $500 \mathrm{~V} / \mathrm{cm}$ is much less than the laser linewidth and more than $10 \%$ suppression has been observed for this state, as shown in Figure 2, which indicates that the polarization of atoms under an electric field may contribute to the excitation suppression. This topic is still under investigation.
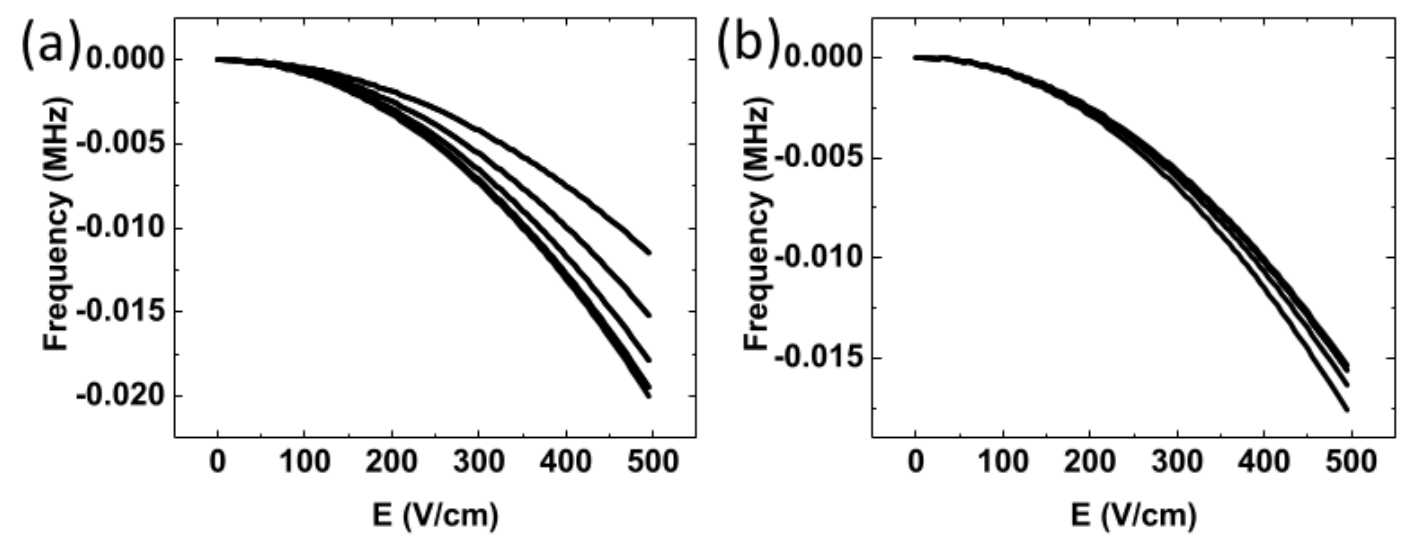

Figure 3. (a) the frequency shift between the $5^{2} P_{3 / 2} \mathrm{~F}=4$ state and the $5^{2} S_{1 / 2} \mathrm{~F}=3$ state under an electric field, or the Stark effect of the trapping transition of the ${ }^{85} \mathrm{Rb}$ atoms; (b) the Frequency shift between the $5^{2} P_{3 / 2} \mathrm{~F}=3$ state and the $5^{2} S_{1 / 2} \mathrm{~F}=2$ state under an electric field, or the Stark effect of the repump transition of the ${ }^{85} \mathrm{Rb}$ atoms. In both plots, we shift the zero field energy difference to zero.

\section{Coulomb Blockade}

\subsection{Theory}

To study Coulomb blockade, we ionize some atoms in the MOT, and we examine the population suppression. Here is a brief theory. We calculate the frequency shift of the energy level of one atom in the presence of an ion. The energy shifts of an atom are caused by dipole-ion or dipole-charge interactions. Here, we treat the atom under the Coulomb field as an induced dipole. A valence electron and an ion core are the two poles of an electric dipole. As shown in Figure 4, an atom and a free ion are separated by a distance $\mathrm{R}$. The ion core of the atom is at the origin of the $x, y$, and $z$-axes. The radius of this atom is $r$. The distance between the electron of the atom and the free ion is $R^{\prime}$. The angle $\theta$ is the angle between $\mathrm{R}$ and $\mathrm{r}$. In this article, we assume the radius of the atoms, $r$, is much smaller than the spacing between the two ions, R. We apply the Born-Oppenheimer approximation, that is, the wave functions of the ion cores and electrons can be separated. We further assume that the ions are at rest. Then, the Hamiltonian of the three-body system shown in Figure 4 can be written as:

$$
H=-\frac{\hbar^{2}}{2 m} \nabla^{2}+V,
$$


where $m$ is the effective electron mass; $\hbar=\frac{h}{2 \pi}$, and $h$ is the Planck's constant. The potential energy V in Equation (3) can be written as:

$$
V=\frac{e^{2}}{4 \pi \epsilon_{0}}\left(-\frac{1}{r}-\frac{1}{R^{\prime}}+\frac{1}{R}\right),
$$

where $e$ is the electron charge, and $\epsilon_{0}$ is the permittivity of free space. Figure 4 shows that

$$
\frac{1}{R^{\prime}}=\frac{1}{\sqrt{(R-r \operatorname{Cos}(\theta))^{2}+r^{2} \operatorname{Sin}^{2}(\theta)}} .
$$

The above equation can be also derived from the law of Cosine. If $R>>r$, we can do the Taylor expansion for $\frac{1}{R^{\prime}}$ at $\mathrm{R}$. The first few orders are shown below:

$$
\frac{1}{R^{\prime}}=\frac{1}{R}+\frac{r \operatorname{Cos}(\theta)}{R^{2}}+\frac{r^{2}\left(3 \operatorname{Cos}^{2}(\theta)-1\right)}{2 R^{3}}+\cdots \cdots
$$

If we keep the first two terms, the Hamiltonian can be rewritten as

$$
H \approx-\frac{\hbar^{2}}{2 m} \nabla^{2}+\frac{e^{2}}{4 \pi \epsilon_{0}}\left(-\frac{1}{r}-\frac{1}{R}-\frac{r \operatorname{Cos}(\theta)}{R^{2}}+\frac{1}{R}\right),
$$

or

$$
H \approx-\frac{\hbar^{2}}{2 m} \nabla^{2}-\frac{e^{2}}{4 \pi \epsilon_{0}} \frac{1}{r}-\frac{e^{2}}{4 \pi \epsilon_{0}} \frac{r \operatorname{Cos}(\theta)}{R^{2}} .
$$

Notice that the combination of the first two terms is the Hamiltonian of a Hydrogen atom, and the last term is the perturbation or the dipole-charge interaction potential energy, $V_{d c}$,

$$
V_{d c}=-\frac{e^{2}}{4 \pi \epsilon_{0}} \frac{r \operatorname{Cos}(\theta)}{R^{2}}
$$

If the higher-order terms are included, the multipole-charge interactions, $V_{m c}$, are

$$
V_{m c}=-\frac{e^{2}}{4 \pi \epsilon_{0} R^{m+1}} r^{m} C_{m, 0}
$$

where $m$ in an integer. For example, if $m=1$, it is dipole-charge interactions, as shown in Equation (9). $C_{m, 0}$ is an $m^{\text {th }}$ order sphereical tensor [25]. In this article, we focus on the dipole-charge interactions, which is the dominant interaction in the case that we considered.

This Hamiltonian can be solved by diagonalizing the matrix composed of the eigenfunctions of a Hydrogen atom. In this article, we simplify this problem by taking advantage of the known Stark effect calculations, as shown in Equation (1) [24]. Since $r \operatorname{Cos}(\theta)=z$, we just need to replace the external electric field by the Coulomb field: $\frac{e}{4 \pi \epsilon_{0} R^{2}}$. More specifically, the interaction energy between an ion and an atom can be written as:

$$
H_{\text {ion-dipole }}=\left(\frac{e}{4 \pi \epsilon_{0} R^{2}}\right)^{2}\left[-\frac{1}{2} \alpha_{0}-\frac{1}{2} \alpha_{2} \frac{3 J_{z}^{2}-J(J+1)}{J(2 J-1)}\right] .
$$

We use the same scalar and tensor polarizabilities, $\alpha_{0}$ and $\alpha_{2}$, described in the DC field suppression session. 


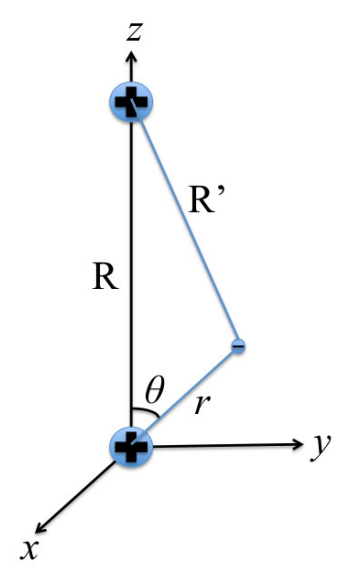

Figure 4. An ion at the top and an atom at the origin of the Cartesian coordinate are separated by a distance R.

\subsection{Experiment}

Figure 5 shows the schematic plot of this experiment. Here, we use a UV laser to ionize atoms because a UV laser, with a greater photon energy compared to a visible photon, can create electrons and ions with greater kinetic energies. It has been shown in previous literature [26] that the electrons will leave the MOT first and leave the ions behind, which is caused by the momentum conservation. Specifically, the electrons will move a lot faster than the ions once they are ionized since the electron mass is much smaller than the mass of the ion core. Therefore, using a high energy laser, the pure ion environment will be achieved faster due to the greater kinetic energy of the electrons, when the system reaches the equilibrium. A continuous UV diode laser produces a UV laser light at $405 \mathrm{~nm}$. The power of this UV laser is about $100 \mathrm{~mW}$, and the temperature of this UV laser is not stabilized. We would expect that the wavelength will drift slightly over time. However, the temperature is relatively stable at the time that the data were taken, since we typically wait for some time to let the system stabilize. The laser beam will then pass through a mechanical shutter. The shutter's turning on speed is $10 \mathrm{~ms}$. After passing a converging lens, the laser beam is focused on the MOT (Magneto-Optical Trap) held in a vacuum chamber. The MOT size is slightly over $3 \mathrm{~mm}$ in diameter. The size of the beam varies over the MOT area. The spacing between neighboring atoms is estimated to be about $2 \mu \mathrm{m}$.

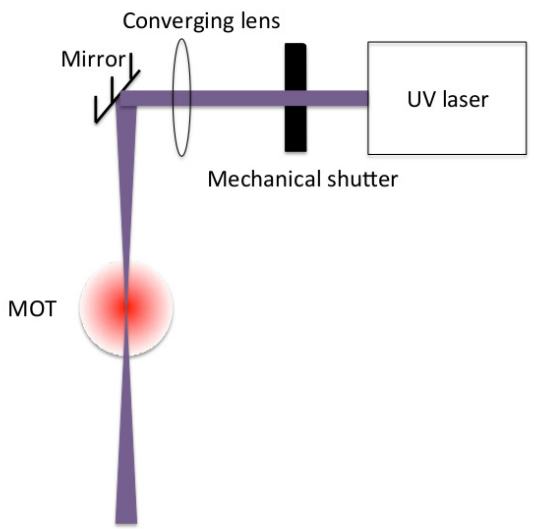

Figure 5. The schematic plot of this experiment. 


\subsection{Results and Discussion}

Figure 6 shows the plasma suppression data and images. Figure $6 b, c$ show two images taken without the ions (Figure 6b,c). It is shown that fewer atoms are excited with the ions, or excitation suppression is observed. The vertical axis is the relative number of cold atoms, and the horizontal axis is the time. It is shown that the percent suppression is about $15 \%$. In this experiment, the $405 \mathrm{~nm} \mathrm{UV}$ light is turned on at about $29 \mathrm{~s}$ by opening the shutter in front of the UV laser (this set of data is one portion of a larger set of data, and the trend reported here is repeatable). The images are continuously taken at a $5 \mathrm{~Hz}$ repetition rate. Since the data were continuously taken, no error bars are given in this plot. The time is estimated from the image taken rate. This rate is confirmed by the shutter switching rate. For example, if the shutter switching rate is set at $0.1 \mathrm{~Hz}$, then within one shutter switching cycle, 50 images will be taken. It is shown that the MOT density decreases when the UV laser was shined on the MOT. The MOT unloading time by blocking one of the trapping beams is much less than $0.2 \mathrm{~s}$. In other words, the MOT is gone within $0.2 \mathrm{~s}$ after blocking one of the trapping beams. We have tried similar experiments by exciting atoms right above the ionization limit, and similar excitation suppression results were obtained.
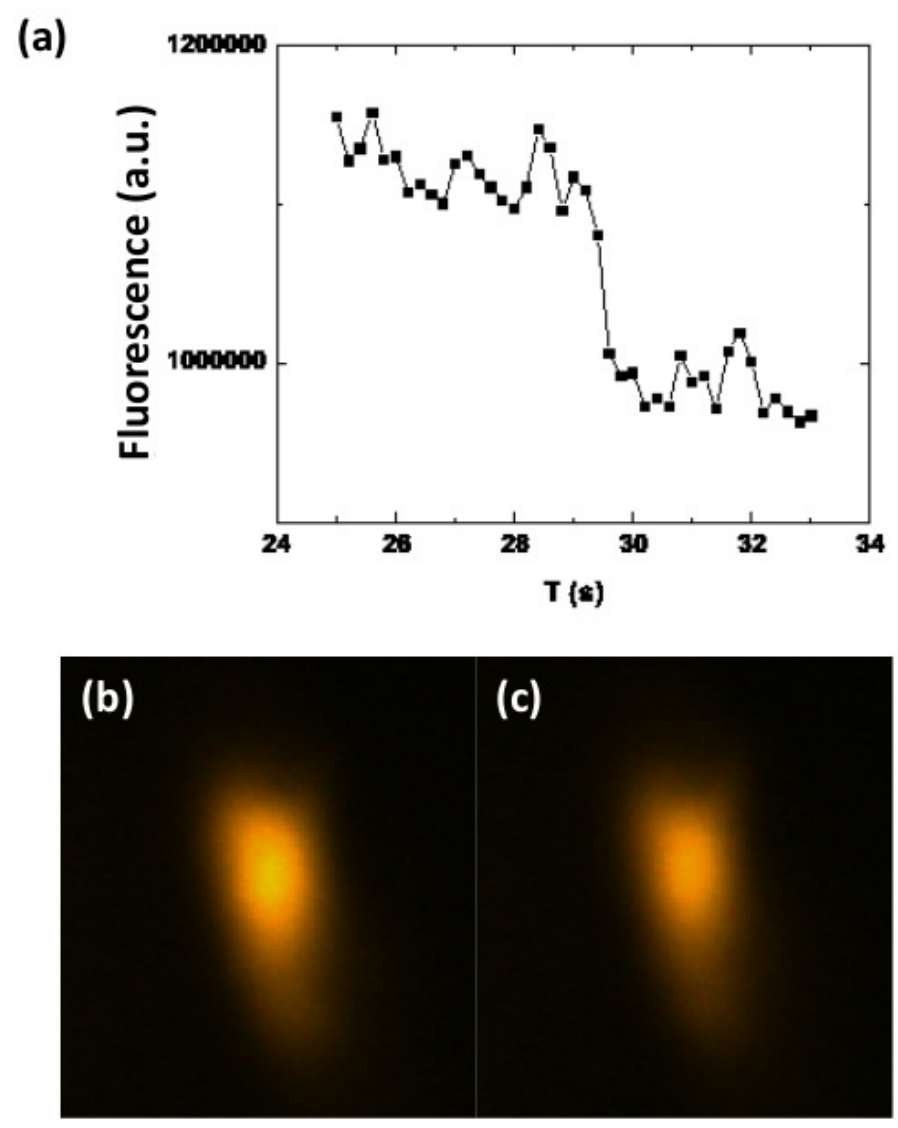

Figure 6. (a) the relative number of atoms as a function of time. The UV light was turned on at about $29 \mathrm{~s}$, as shown in this plot. (b) The image was taken at about $28.4 \mathrm{~s}$, as shown in (a). (c) The image was taken at about $32.4 \mathrm{~s}$, as shown in (a). 
Figure 7 shows the energy shift of the trapping transition and repump transition under an electric field produced by an ion, as shown in Figure 4. For example, Figure 7a is calculated in the following way. We first calculate the energy difference between the $5^{2} P_{3 / 2} \mathrm{~F}=4$ level and the $5^{2} S_{1 / 2} \mathrm{~F}=3$ level under an ion field. We then subtract this energy difference by the energy difference between those two states under zero electric field. Another way of expressing this is

$$
E=\left(E_{5^{2} P_{3 / 2}, F=4, \text { ion }}-E_{5^{2} S_{1 / 2}, F=3, \text { ion }}\right)-\left(E_{5^{2} P_{3 / 2},=4}-E_{5^{2} S_{1 / 2}, F=3}\right) .
$$

For example, the difference between $E_{5^{2} P_{3 / 2}, F=4, i o n}$ and $E_{5^{2} P_{3 / 2}, F=4}$ is that $E_{5^{2} P_{3 / 2}, F=4, i o n}$ is the energy under an ion field, while $E_{5^{2} P_{3 / 2}, F=4}$ is the energy of the same level under zero field. Similar to the DC field case, the different levels correspond to different $F_{z}$ states. It is shown that, at about $0.8 \mu \mathrm{m}$, the maximum frequency shift caused by the ion field is about $0.5 \mathrm{MHz}$, which is comparable to the laser linewidth that we used for this experiment. Considering the non-uniform distribution, for example, a Gaussian distribution, of the atoms in the MOT, the theory is reasonable to explain the $15 \%$ suppression of the experimental data. Here, we assume that the $\frac{1}{R^{4}}$ dependence dominates, and other $R$ dependence is still under investigation. In addition to the suppression caused by ionized atoms, the population will be slightly reduced by ionization, which causes the slight reduction in the fluorescence.
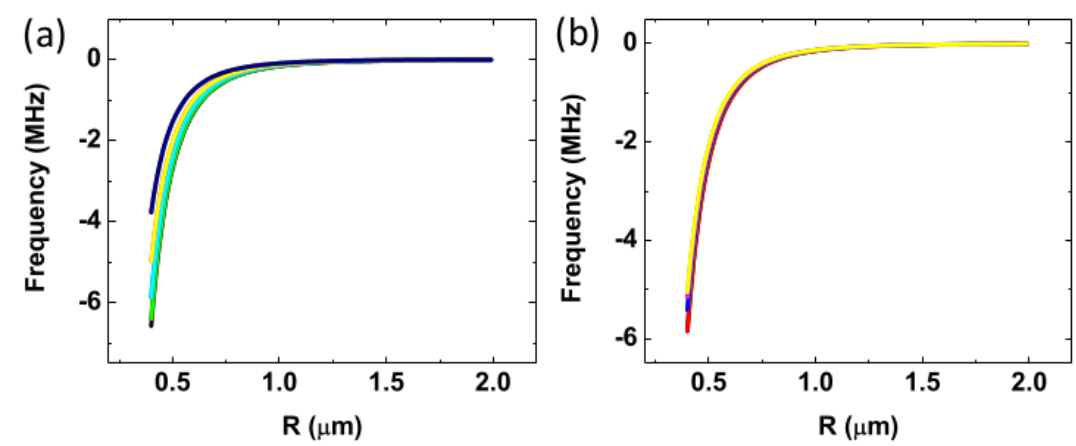

Figure 7. (a) the trapping transition shift due to ions; (b) the repump transition shift due to ions.

\section{Conclusions}

This work provides a detailed study of excitation suppression in low-lying atomic states. We first considered an external DC electric field to suppress the ground state excitation. We subsequently studied the suppression caused by a Coulomb interaction with a neighboring ion. The frequency shift caused by the Coulomb field from an ion is calculated by taking advantage of the known Stark effect calculations. We have found that the frequency shift is about half a MHz at $\mathrm{R}=0.8 \mu \mathrm{m}$, where $\mathrm{R}$ is the distance between the ion and the atom. Moreover, we have experimentally observed $15 \%$ excitation suppression caused by the ions produced by a UV laser, which is explained by our theoretical model assuming an average internuclear spacing of $2 \mu \mathrm{m}$.

Author Contributions: All authors contributed equally to this project; funding acquisition, J.H. All authors have read and agreed to the published version of the manuscript.

Funding: This research was funded by the Air Force Office of Scientific Research (AFOSR), Grant No. FA9550-20-1-0009 and FA9550-20-1-0099".

Acknowledgments: It is a pleasure to acknowledge very helpful discussions with Tom F. Gallagher, Gao Bo, and Justin Sanders. We would like to acknowledge the current support from the US Air Force Office of Scientific Research (AFOSR), the University of South Alabama Faculty Development Council (USAFDC), and a previous award from the Army Research Office (ARO). Juliet would like to thank the SURF program from the University of South Alabama and the Helminger award from the physics department at the University of South Alabama.

Conflicts of Interest: The authors declare no conflict of interest. 


\section{References}

1. Grabert, H.; Devoret, M.H. Single Charge Tunneling: Coulomb Blockade Phenomena in Nanostructures; Plenum Press: New York, NY, USA, 1992.

2. Mecklenburg, M.; Kubala, B.; Ankerhold, J. Thermopower and dynamical Coulomb blockade in nonclassical environments. Phys. Rev. B 2017, 96, 155405. [CrossRef]

3. Pikulin, D.; Flensberg, K.; Glazman, I.L.; Houzet, M.; Lutchyn, R.M. Coulomb Blockade of a Nearly Open Majorana Island. Phys. Rev. Lett. 2019, 122, 016801. [CrossRef] [PubMed]

4. Cote, R. From Classical Mobility to Hopping Conductivity: Charge Hopping in an Ultracold Gas. Phys. Rev. Lett. 2000, 85, 5316. [CrossRef] [PubMed]

5. Engel, F.; Dieterle, T.; Schmid, T.; Tomschitz, C.; Veit, C.; Zuber, N.; Low, R.; Pfau, T.; Meinert, F. Observation of Rydberg Blockade Induced by a Single Ion. Phys. Rev. Lett. 2018, 121, 193401. [CrossRef]

6. Raimond, J.; Vitrant, G.; Haroche, S. Spectral line broadening due to the interaction between very excited atoms: 'the dense Rydberg gas'. J. Phys. B 1981, 14, L655. [CrossRef]

7. Jaksch, D.; Cirac, J.I.; Zoller, P.; Rolston, S.L.; Cote, R.; Lukin, M.D. Fast Quantum Gates for Neutral Atoms. Phys. Rev. Lett. 2000, 85, 2208. [CrossRef]

8. Lukin, M.D.; Fleischhauer, M.; Cote, R.; Duan, L.M.; Jaksch, D.; Cirac, J.I.; Zoller, P. Dipole Blockade and Quantum Information Processing in Mesoscopic Atomic Ensembles. Phys. Rev. Lett. 2001, 87, 037901. [CrossRef]

9. Afrousheh, K.; Bohlouli-Zanjani, P.; Vagale, D.; Mugford, A.; Fedorov, M.; Martin, J.D.D. Spectroscopic Observation of Resonant Electric Dipole-Dipole Interactions between Cold Rydberg Atoms. Phys. Rev. Lett. 2004, 93, 233001. [CrossRef]

10. Tong, D.; Farooqi, S.M.; Stanojevic, J.; Krishnan, S.; Zhang, Y.P.; Cote, R.; Eyler, E.E.; Gould, P.L. Local Blockade of Rydberg Excitation in an Ultracold Gas. Phys. Rev. Lett. 2004, 93, 063001. [CrossRef]

11. Singer, K.; Reetz-Lamour, M.; Amthor, T.; Gustavo Marcassa, L.; Weidemuller, M. Suppression of Excitation and Spectral Broadening Induced by Interactions in a Cold Gas of Rydberg Atoms. Phys. Rev. Lett. 2004, 93, 163001. [CrossRef]

12. Heidemann, R.; Raitzsch, U.; Bendkowsky, V.; Butscher, B.; Low, R.; Santos, L.; Pfau, T. Evidence for Coherent Collective Rydberg Excitation in the Strong Blockade Regime. Phys. Rev. Lett. 2007, 99, 163601. [CrossRef] [PubMed]

13. Liebisch, T.C.; Reinhard, A.; Berman, P.R.; Raithel, G. Atom Counting Statistics in Ensembles of Interacting Rydberg Atoms. Phys. Rev. Lett. 2005, 95, 253002. [CrossRef] [PubMed]

14. Urban, E.; Johnson, T.A.; Henage, T.; Isenhower, L.; Yavuz, D.D.; Walker, T.G.; Saffman, M. Observation of Rydberg blockade between two atoms. Nature Phys. 2009, 5, 110. [CrossRef]

15. Gaetan, A.; Miroshnychenko, Y.; Wilk, T.; Chotia, A.; Viteau, M.; Comparat, D.; Pillet, P.; Browaeys, A.; Grangier, P. Observation of collective excitation of two individual atoms in the Rydberg blockade regime. Nature Phys. 2009, 5, 115. [CrossRef]

16. Gallagher, T.F. Rydberg Atoms; Cambridge University Press: Cambridge, UK, 1994.

17. Stebbings, R.F.; Dunning, F.B. Rydberg States of Atoms and Molecules; Cambridge University Press: Cambridge, UK, 1983.

18. Li, W.; Noel, M.W.; Robinson, M.P.; Tanner, P.J.; Gallagher, T.F.; Comparat, D.; Tolra, B.L.; Vanhaecke, N.; Vogt, T.; Zahzam, N.; et al. Evolution dynamics of a dense frozen Rydberg gas to plasma. Phys. Rev. A 2004, 70, 042713. [CrossRef]

19. Killian, T.C.; Kulin, S.; Bergeson, S.D.; Orozco, L.A.; Orzel, C.; Rolston, S.L. Creation of an Ultracold Neutral Plasma. Phys. Rev. Lett. 1999, 83, 4776. [CrossRef]

20. Gallagher, T.F.; Pillet, P.; Robinson, M.P.; Laburthe-Tolra, B.; Noel, M.W. Back and forth between Rydberg atoms and ultracold plasmas. J. Opt. Soc. Am. B 2003, 20, 1091. [CrossRef]

21. Tanner, P.J.; Han, J.; Shuman, E.S.; Gallagher, T.F. Many-Body Ionization in a Frozen Rydberg Gas. Phys. Rev. Lett. 2008, 100, 043002. [CrossRef]

22. Han, J.; Hutcherson, L.; Deshmukh, G.; Umstead, M.; Hu, A.; Lee, Y.; Bai, Z.; Mitchell, J. A magneto-optical trap created by the 2nd-order external cavity diode lasers. arXiv 2018, arXiv:1812.00997. 
23. Li, W.; Mourachko, I.; Noel, M.W.; Gallagher, T.F. Millimeter-wave spectroscopy of cold Rb Rydberg atoms in a magneto-optical trap: Quantum defects of the ns, np, and nd series. Phys. Rev. A 2003, 67, 052502. [CrossRef]

24. Adam Steck, D. Rubidium 85 D Line Data. Available online: http://steck.us/alkalidata (accessed on 30 April 2009).

25. Edmonds, A.R. Angular Momentum in Quantum Mechanics; Princeton University Press: Princeton, NJ, USA, 1957.

26. Li, W.; Tanner, J.P.; Gallagher, T.F. Dipole-Dipole Excitation and Ionization in an Ultracold Gas of Rydberg Atoms. Phys. Rev. Lett. 2005, 94, 173001. [CrossRef] [PubMed]

(C) 2020 by the authors. Licensee MDPI, Basel, Switzerland. This article is an open access article distributed under the terms and conditions of the Creative Commons Attribution (CC BY) license (http://creativecommons.org/licenses/by/4.0/). 